PoS $\quad \begin{aligned} & \text { PROCEEDINGS } \\ & \text { OF SCIENCE }\end{aligned}$

\title{
Summary of recent progress in searches for additional Higgs bosons
}

\author{
Martin Flechl* \\ Institute of High Energy Physics, \\ Austrian Academy of Sciences \\ E-mail: martin.flechl@cern.ch
}

\begin{abstract}
There has been a tremendous increase of sensitivity in searches for charged Higgs bosons and additional neutral Higgs bosons since the last CHARGED workshop in 2016. We will review recent experimental and theory developments as presented at CHARGED2018, and conclude with future prospects for the field.
\end{abstract}

Prospects for Charged Higgs Discovery at Colliders - CHARGED2018

25-28 September 2018

Uppsala, Sweden

\footnotetext{
* Speaker.
} 


\section{Introduction}

Additional Higgs bosons $\mathrm{H}^{ \pm}$appear in many extensions of the standard model (SM), in particular when adding additional doublets or triplets to its scalar sector. Typically, the focus is on 2-Higgs-doublet models (2HDMs) including the special case of the Higgs sector of the minimal supersymmetric extension of the standard model (MSSM). In the MSSM, the dominant production mode for a charged Higgs boson is in top quark decays if kinematically allowed, or in association with a top quark otherwise. Neutral Higgs bosons are dominantly produced via gluon fusion (for low values of $\tan \beta$, the ratio of the vacuum expectation values of the two Higgs doublets) and in association with bottom quarks (intermediate and high $\tan \beta$ ). However, non-standard production and decay modes may become dominant in other 2HDMs or for specific relations between the Higgs boson masses, allowing e.g. Higgs-to-Higgs decays. Other beyond-the-standard-model (BSM) Higgs sectors offer an even larger variety: for example, the next-to-MSSM (NMSSM) predicts two neutral Higgs bosons in addition to the MSSM which could be relatively light without violating existing bounds; and models with Higgs triplets predict among others doubly-charged Higgs bosons.

\section{Charged Higgs bosons}
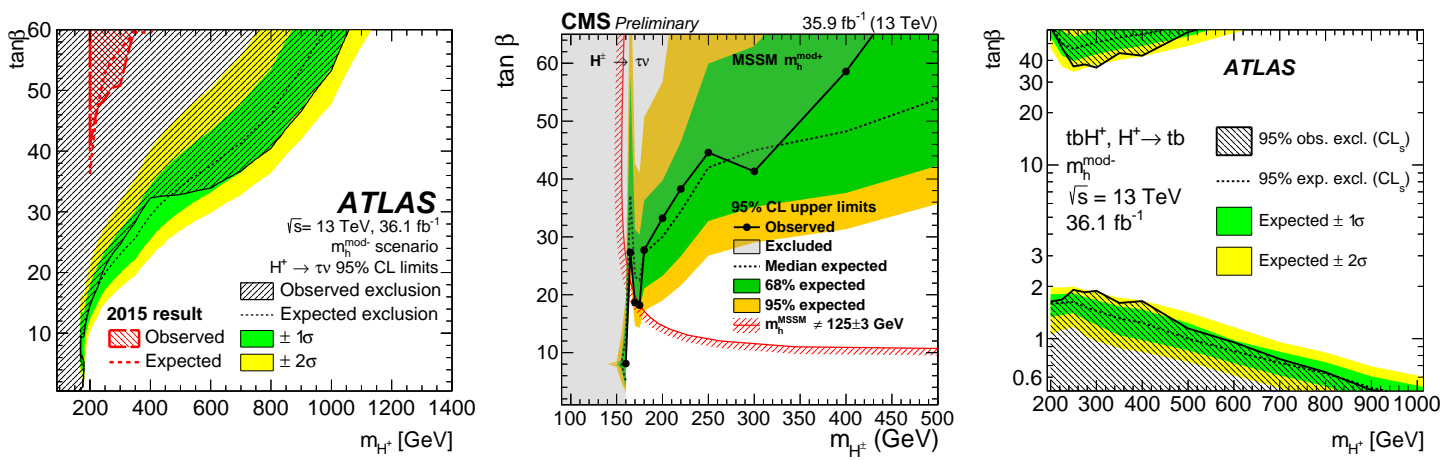

Figure 1: 95\% CL limits for the MSSM interpretation of the search for $H^{ \pm} \rightarrow \tau \nu$ by ATLAS [7] (left) and CMS [8] (middle), and of $H^{ \pm} \rightarrow t b$ by ATLAS [9] (right).

Charged Higgs boson $\left(H^{ \pm}\right)$searches are primarily motivated by the MSSM but the results are also given in a largely model-independent way, i.e. in terms of cross section limits. There is also an increasing number of non-MSSM searches, e.g. for charged Higgs boson production in vector boson fusion [1]. For masses below the top quark mass, a charged Higgs boson would be dominantly produced in top quark decays. Therefore, the production cross section is proportional to the top quark pair production times the branching ratio $\mathrm{t} \rightarrow \mathrm{H}^{+} \mathrm{b}$. The dominant mode of $\mathrm{H}^{ \pm}$ production for $m_{\mathrm{H}^{ \pm}}>m_{\mathrm{t}}$ in a $2 \mathrm{HDM}$ is via the process $\mathrm{pp} \rightarrow \mathrm{tH}^{ \pm}+X[2,3,4,5,6]$. Recent progress on theory side has led to the first consistent NLO calculations also for the so-called intermediatemass region [10], i.e. the region where the contributions with and without intermediate on-shell top quarks are of similar size. This region is of particular interest because MSSM models where 
the heavy neutral scalar has a mass of $125 \mathrm{GeV}$ and the light Higgs boson can act as mediator to dark matter typically have a charged Higgs boson mass in this region.

In the MSSM, the most sensitive $H^{ \pm}$decay mode is to a $\tau$ lepton and a neutrino [7, 8], except for very low values of $\tan \beta$. If kinematically allowed, decays to $t b$ are typically similarly abundant, however, reconstructing such events is experimentally even more challenging [9]. An MSSM interpretation of the obtained limits is given in Fig. 1. Previous limits have been extended from several hundreds of $\mathrm{GeV}$ to one $\mathrm{TeV}$ (for the same value of $\tan \beta$ ) in the last two years, marking tremendous progress since the last workshop. These results also mark the first results for the intermediate-mass region.

Several other ways to search for charged Higgs bosons have been proposed to the experimental collaborations at this workshop. Examples are charged Higgs boson decays to $\mathrm{W} \gamma$ [11], $H^{ \pm}$production in $c \bar{s}$ fusion in a 3HDM [12], or charged Higgs boson decays to neutralinos and charginos [13].

\section{Doubly-charged Higgs bosons}

Doubly-charged Higgs bosons $H^{ \pm \pm}$are for example predicted by models with Higgs triplets such as left-right symmetric models or the Georgi-Machacek model, and the Zee-Babu model which only adds two singlets to the SM. Variants of these models can serve to explain the tiny observed neutrino masses or to restore parity symmetry in weak interactions at high energy. Models with triplets do not automatically lead to the observed value of $\rho \equiv \frac{M_{W}^{2}}{\cos ^{2} \theta_{W}^{2} M_{Z}^{2}}$ close to unity, as models with only additional singlets and doublets do, and are thus more or less severely constrained.

For several models, the main $H^{ \pm \pm}$production mechanism at the LHC is via $\mathrm{q} \overline{\mathrm{q}} \rightarrow \mathrm{Z} / \gamma^{*} \rightarrow$ $\mathrm{H}^{++} \mathrm{H}^{--}$or $\mathrm{q} \overline{\mathrm{q}} \rightarrow \mathrm{W} \rightarrow \mathrm{H}^{++} \mathrm{H}^{-}$. Searches at the LHC focus at $H^{ \pm \pm}$decays to leptons $[14,15]$ or W bosons [16]. The $H^{ \pm \pm}$coupling to leptons is typically not proportional to the lepton mass and the focus is thus on light leptons which can be handled more easily experimentally. The result of some of the LHC searches are illustrated in Fig. 2. Depending on models and assumed branching ratios, typically $H^{ \pm \pm}$below $500 \mathrm{GeV}$ to $900 \mathrm{GeV}$ are excluded.
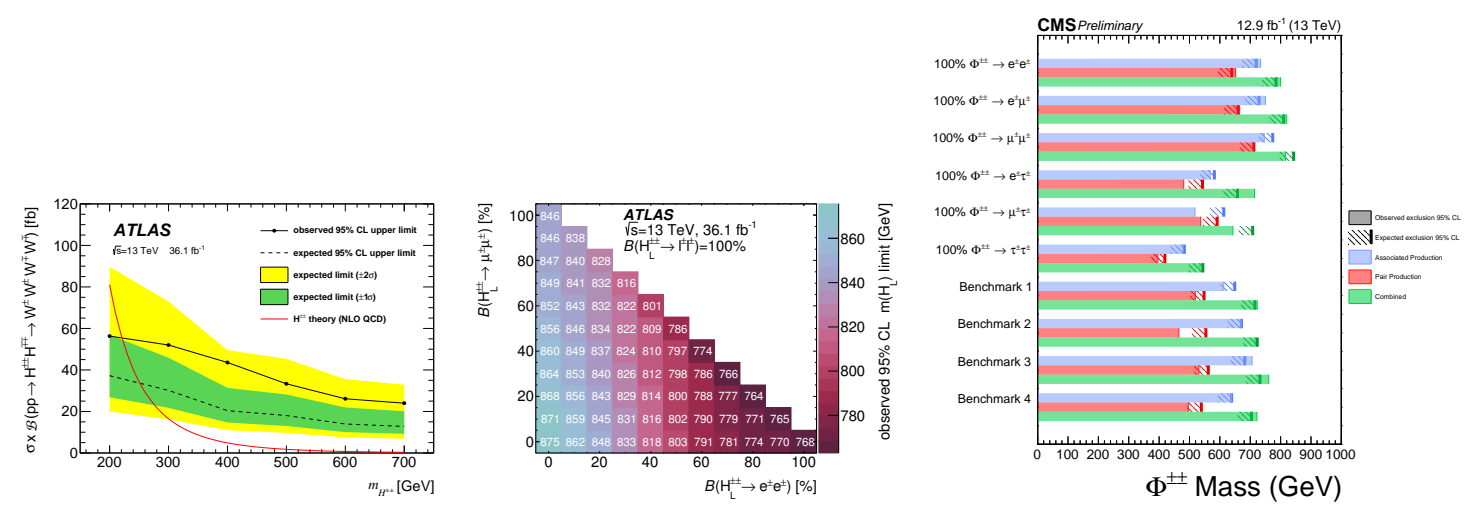

Figure 2: Limits from searches for doubly charged Higgs bosons in pair production and decays to leptons by ATLAS [14] (left) and CMS [15] (middle), as well as in decays to W bosons by ATLAS [16] (right). 


\section{Additional neutral Higgs bosons}

Additional neutral Higgs bosons are the ingredient of virtually every model with an extended Higgs sector. The main motivation for searches for these particles is traditionally the MSSM; however, the portfolio has been significantly extended since the LHC start. In addition, the MSSM searches can be reinterpreted in the context of other models. The main LHC production mode for neutral MSSM Higgs bosons are associated production with $\mathrm{b}$ quarks (intermediate and high $\tan \beta$ ) as well as gluon fusion (low $\tan \beta$ ). For all but very low $\tan \beta$ values, decays to $\tau$ leptons are the most sensitive experimental probe $[17,18]$ with decays to bottom quarks adding additional sensitivity [19], as shown in Fig. 3. Since the last workshop, the excluded region has been significantly extended, reaching up to $1.6 \mathrm{TeV}$ at high $\tan \beta$. At this workshop, adding the investigation of neutral Higgs boson decays to neutralinos or charginos to the portfolio [13] and investigating the impact of CP-violating effects [20] has been proposed.
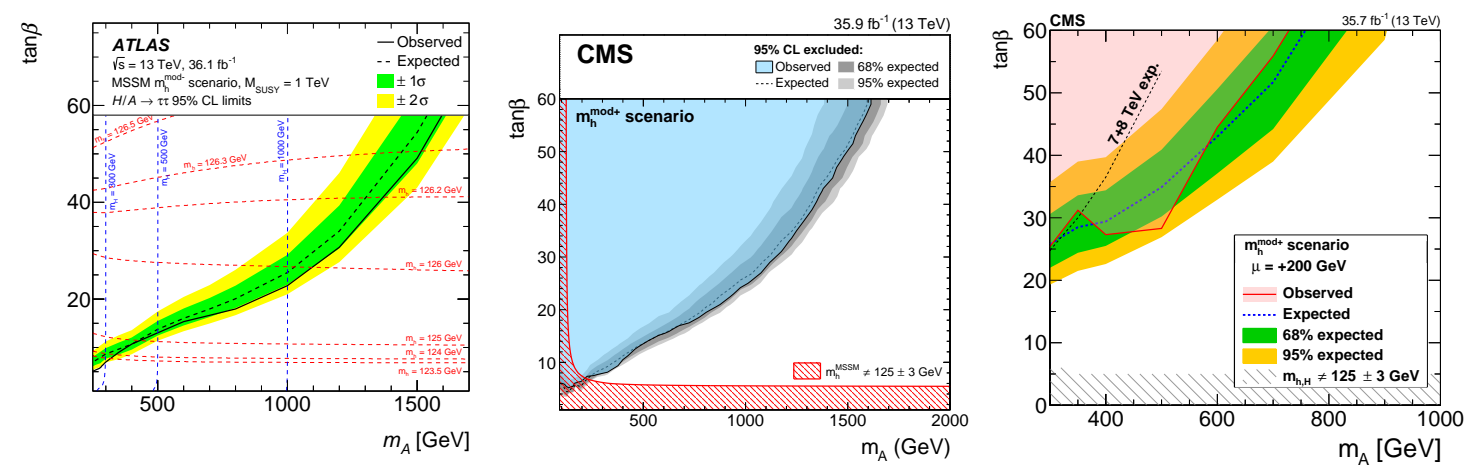

Figure 3: MSSM interpretation of the limits from searches for additional neutral Higgs bosons in $\tau \tau$ decays

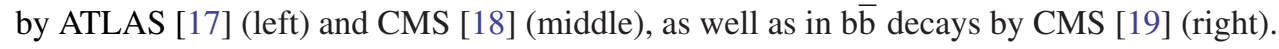

In models with additional Higgs bosons with masses below about $62.5 \mathrm{GeV}$, the $125-\mathrm{GeV}$ Higgs boson can decay to these Higgs bosons, $\mathrm{h} \rightarrow$ aa. Searches for these light Higgs bosons are often motivated by the NMSSM but the results can be interpreted in a generic way. These bosons then dominantly decay to the heaviest particles kinematically allowed, leading to a variety of search modes [21, 22]. The results of two such searches are shown in Fig. 4.

Generically, light Higgs bosons are searched for in $\gamma \gamma$ decays in the mass range $65 \mathrm{GeV}-110$ $\mathrm{GeV}$. Here, a slight excess of events is observed for $m_{\gamma \gamma} \approx 95$ by CMS [23] which is not confirmed by ATLAS [24], see Fig. 5. Similarly, generic Higgs boson searches are extended to high masses up to $4 \mathrm{TeV}$ in the WW [25] and ZZ [26] decay modes. At the highest masses, a cross section as low as $1 \mathrm{fb}$ is excluded, see Fig. 6.

\section{Resonant decays to Higgs bosons}

The SM predicts the production of events with two Higgs bosons of a mass of $125 \mathrm{GeV}$ with a relatively low pp cross section at $\sqrt{s}=13 \mathrm{TeV}$ of about $33 \mathrm{fb}$, including diagrams both with and without vertices with three Higgs bosons. This has not been observed yet at the LHC and the current 

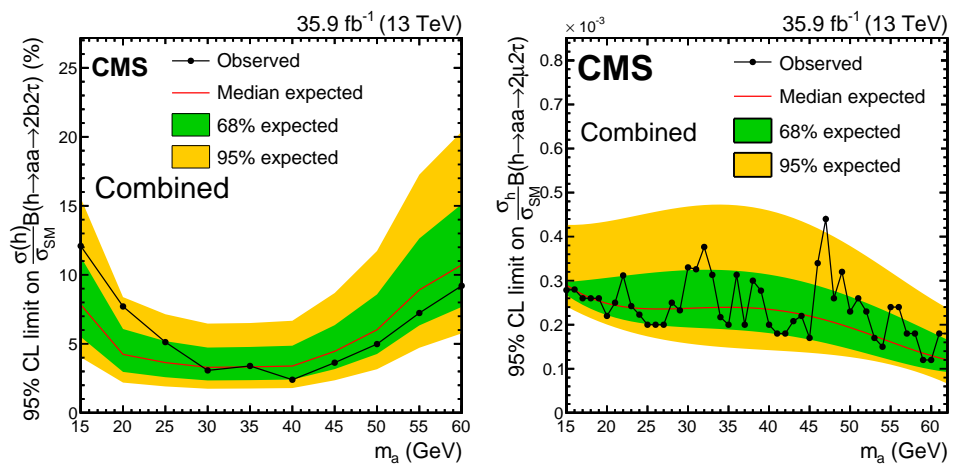

Figure 4: $95 \%$ CL cross section limits on the cross section times branching ratio of $\mathrm{h} \rightarrow$ aa in the $2 \mathrm{~b} 2 \tau$ [21] and $2 \mu 2 \tau$ [22] final states.
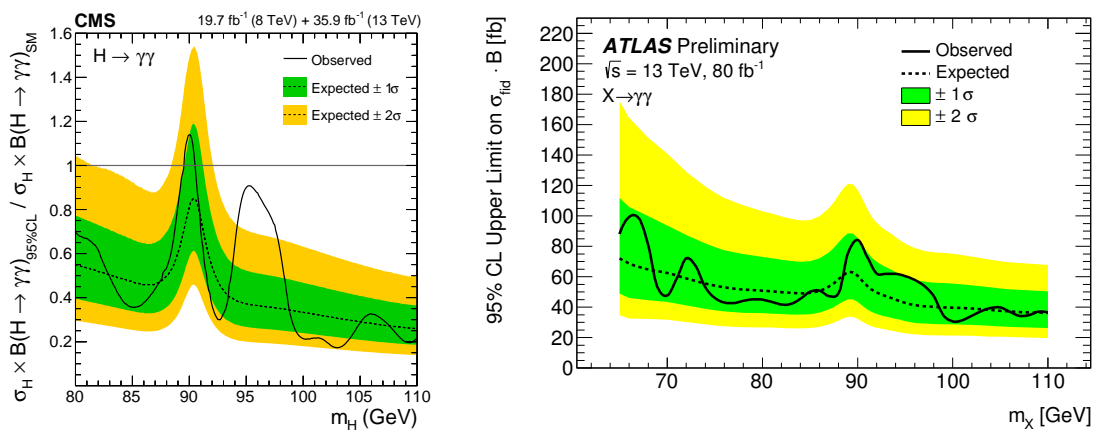

Figure 5: 95\% CL cross section limits on light Higgs bosons in $\gamma \gamma$ decays by CMS [23] (left) and ATLAS [24] (right).

95\% CL limits, in multiples of the expected SM cross section, are 6.7 observed (10.4 expected) for ATLAS [27] and 22.2 observed (12.8 expected) for CMS [28]. An increased rate of HH production could be due to a heavy resonance $\mathrm{X}$ decaying to two Higgs bosons, i.e. $\mathrm{pp} \rightarrow \mathrm{X} \rightarrow \mathrm{HH}$. Both ATLAS and CMS have searched for such resonant decays but found good agreement with the SM expectation. Limits on a scalar resonance decaying to $\mathrm{HH}$, combining several final states, are shown in Fig. 7, and reach from about $1 \mathrm{pb}$ at $m_{\mathrm{X}}=300 \mathrm{GeV}$ down to a few fb at $m_{\mathrm{X}}=3000 \mathrm{GeV}$.

\section{BSM search summary}

For given benchmark scenarios, limits using different final states can be compared which is particularly useful if they have their main sensitivity in different regions of the parameter space. Examples for the MSSM scenarios hMSSM (ATLAS) [29] and $m_{\mathrm{h}}^{\bmod +}$ (CMS) [30] are given in Fig. 8. The most stringent limits are obtained for the hMSSM where an additional neutral Higgs boson with $m_{\mathrm{H}}<500 \mathrm{GeV}$ is excluded (e.g. by using, for each point in parameter space, the observed limit corresponding to the most sensitive analysis there). While analyses of the $\tau \tau$ final state cover the excluded parameter space at intermediate and high $\tan \beta$, decays involving bosons or up-type fermions are needed to close the gap at low $\tan \beta$. 

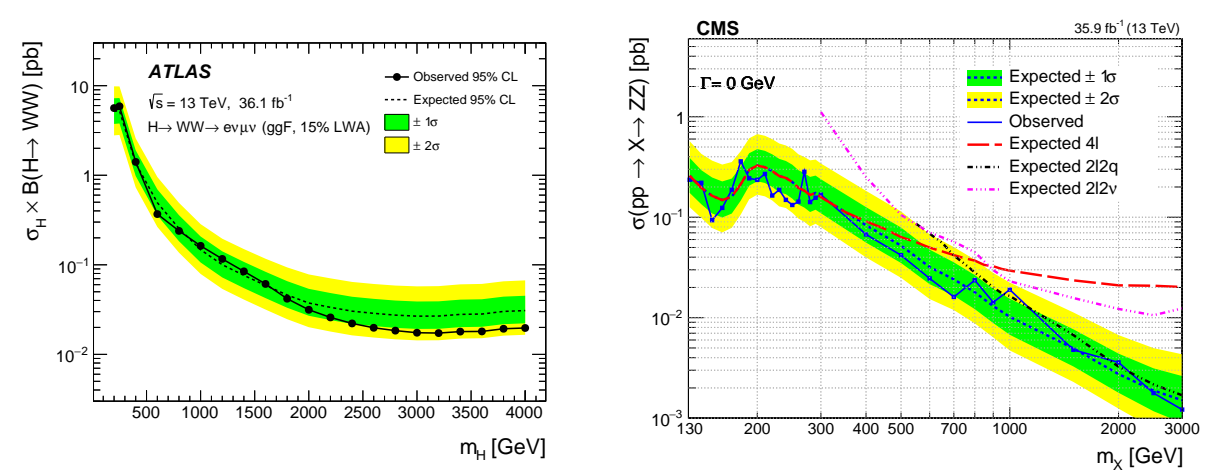

Figure 6: $95 \%$ CL cross section limits on heavy Higgs bosons in VV decays by ATLAS [25] (left) and CMS [26] (right).
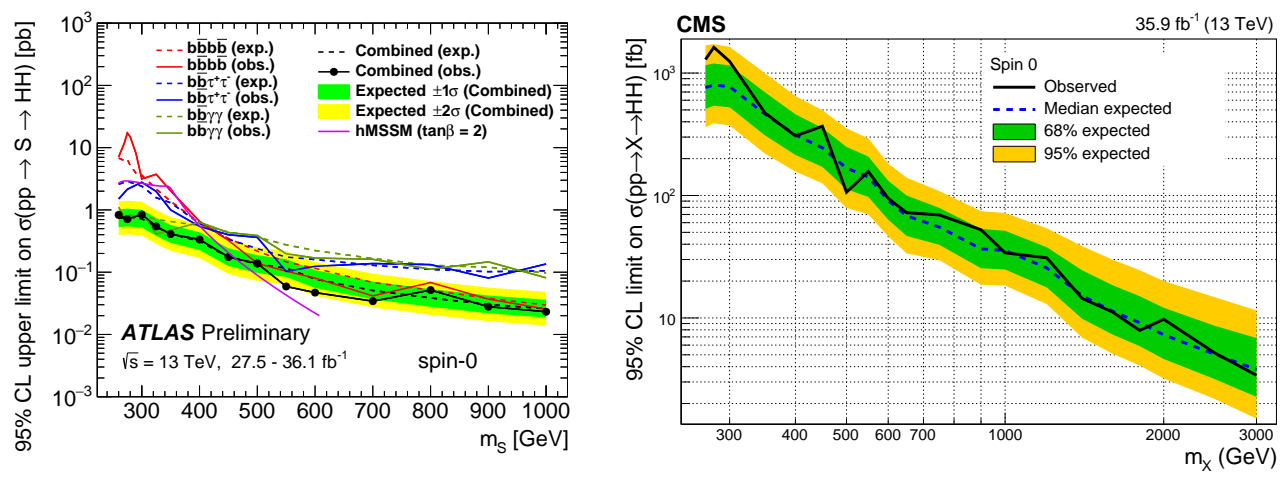

Figure 7: 95\% CL cross section limits on a scalar resonance decaying to two Higgs bosons with $m_{\mathrm{H}}=125$ GeV for ATLAS [27] (left) and CMS [28] (right).

\section{Prospects at future colliders}

Sensitivity projections for neutral MSSM Higgs bosons are shown in Fig. 9 for the LHC and a future pp-collider. While the $\mathrm{LHC}$ will be able to probe a region up to $2 \mathrm{TeV}$ at intermediate and high $\tan \beta$ using $\tau \tau$ decays of the Higgs boson, a future collider will be able to probe the region up to about $5 \mathrm{TeV}$ for all $\tan \beta$, combining the information from different final states.

\section{Summary}

While so far none of the searches for additional Higgs bosons revealed a significant deviation from the SM expectation, the remaining parameter space has been strongly limited in the last two years with an increased amount of analyzed LHC data at $\sqrt{s}=13 \mathrm{TeV}$, and the increasing number of models and final states investigated. The LHC and future colliders will further push these boundaries in the coming decades, having the sensitivity to discover additional Higgs bosons. 

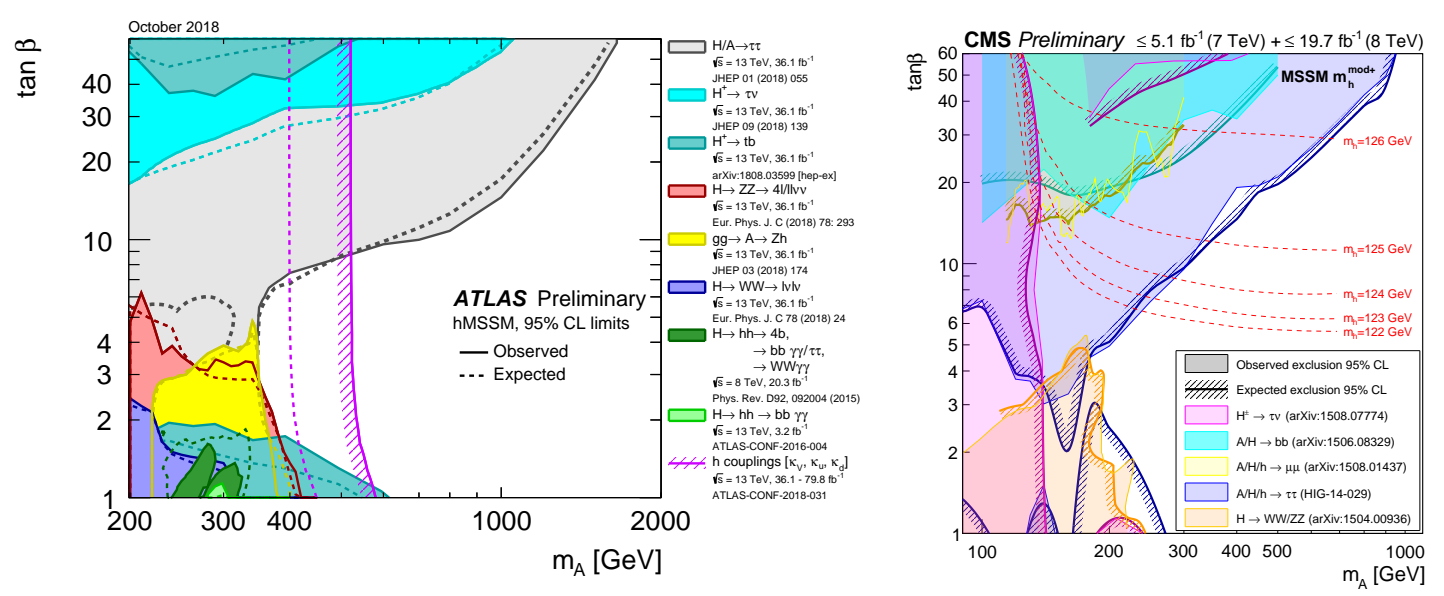

Figure 8: Summary plots of BSM Higgs boson searches for the hMSSM scenario by ATLAS [29] and for the $m_{\mathrm{h}}^{\bmod +}$ scenario by CMS [30].
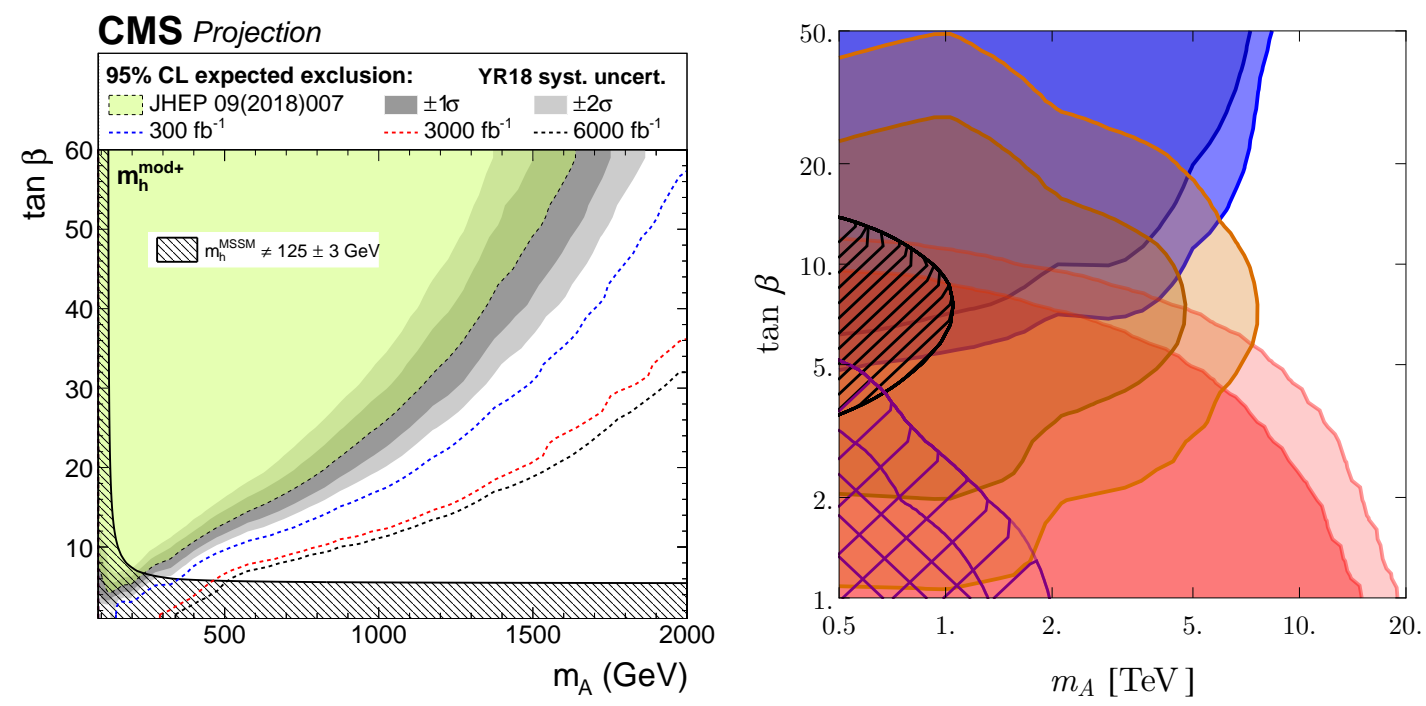

Figure 9: Exclusion sensitivity for neutral heavy Higgs bosons. Left: High-Luminosity LHC [31] reach for $\mathrm{A} \rightarrow \tau \tau$. Right: The low $\tan \beta$ region (red) is covered by $\overline{\mathrm{t}} \mathrm{H}, \mathrm{H} \rightarrow \mathrm{t} \overline{\mathrm{t}}$. The intermediate $\tan \beta$ region (orange) is covered by b-associated production with $\mathrm{H} \rightarrow \mathrm{t} \overline{\mathrm{t}}$ decays. The large $\tan \beta$ region (blue) is covered by b-associated production with $\tau \tau$ decays. The smaller bound (lines) corresponds to 0.3 and $3 \mathrm{ab}^{-1}$, the larger bound (filled regions) to 3 and $30 \mathrm{ab}^{-1}$ at the LHC and a future pp-collider, respectively [32].

\section{References}

[1] CMS Collaboration, Measurement of electroweak WZ production and search for new physics in pp collisions at $\sqrt{s}=13 \mathrm{TeV}$, CMS-PAS-SMP-18-001.

[2] M. Flechl, R. Klees, M. Krämer, M. Spira, M. Ubiali, Improved cross-section predictions for heavy charged Higgs boson production at the LHC, PRD 91 (2015) 075015. 
[3] E.L. Berger, T. Han, J. Jiang, T. Plehn, Associated Production of a Top Quark and a Charged Higgs Boson, PRD 71 (2005) 115012.

[4] S. Dittmaier, M. Krämer, M. Spira, M. Walser, Charged-Higgs-boson production at the LHC: NLO supersymmetric QCD corrections, PRD 83 (2011) 055005

[5] C. Weydert, S. Frixione, M. Herquet, M. Klasen, E. Laenen, T. Plehn, G. Stavenga, C. D. White, Charged Higgs boson production in association with a top quark in MC@NLO, EPJ C67 (2010) 617.

[6] LHC Higgs Cross Section Working Group, Handbook of LHC Higgs cross sections: 4. Deciphering the nature of the Higgs sector, CERN Yellow Reports: Monographs Volume 2/2017.

[7] ATLAS Collaboration, Search for charged Higgs bosons decaying via $H^{+} \rightarrow \tau \nu_{\tau}$ in the $\tau+$ jets and $\tau+$ lepton final states with $36 \mathrm{fb}^{1}$ of pp collision data recorded at $\sqrt{s}=13 \mathrm{TeV}$ with the ATLAS experiment, JHEP 09 (2018) 139.

[8] CMS Collaboration, Search for charged Higgs bosons with the $\mathrm{H}^{ \pm} \rightarrow \tau^{ \pm} v_{\tau}$ decay channel in proton-proton collisions at $\sqrt{s}=13 \mathrm{TeV}$, CMS-PAS-HIG-18-014.

[9] ATLAS Collaboration, Search for charged Higgs bosons decaying into top and bottom quarks at $\sqrt{s}=13$ TeV with the ATLAS detector, JHEP 11 (2018) 085.

[10] C. Degrande, R. Frederix, V. Hirschi, M. Ubiali, M. Wiesemann, M. Zaro, Accurate predictions for charged Higgs production: closing the $m_{H^{ \pm}} \sim m_{t}$ window, arXiv:1607.05291.

[11] H. E. Logan, Y. Wu, Searching for the W $\gamma$ decay of a charged Higgs boson, JHEP 11 (2018) 121.

[12] R. Pasechnik, Heavy charged scalars from cs fusion: A generic search strategy applied to a 3 HDM with $\mathrm{U}(1) \times \mathrm{U}(1)$ family symmetry, JHEP 03 (2018) 024

[13] H. Bahl et. al., MSSM Higgs Boson Searches at the LHC: Benchmark Scenarios for Run 2 and Beyond, arXiv:1808.07542.

[14] ATLAS Collaboration, Search for doubly charged Higgs boson production in multi-lepton final states with the ATLAS detector using proton-proton collisions at $\sqrt{s}=13 \mathrm{TeV}$, EPJ C78 (2018) 199.

[15] CMS Collaboration, A search for doubly-charged Higgs boson production in three and four lepton final states at $\sqrt{s}=13 \mathrm{TeV}$, CMS-PAS-HIG-16-036.

[16] ATLAS Collaboration, Search for doubly charged scalar bosons decaying into same-sign W boson pairs with the ATLAS detector $\sqrt{s}=13 \mathrm{TeV}$, arXiv:1808.01899, submitted to EPJ C.

[17] ATLAS Collaboration, Search for additional heavy neutral Higgs and gauge bosons in the ditau final state produced in $36 \mathrm{fb}^{-1}$ of pp collisions at $\sqrt{\mathrm{s}}=13 \mathrm{TeV}$ with the ATLAS detector, JHEP 01 (2018) 055.

[18] CMS Collaboration, Search for additional neutral MSSM Higgs bosons in the $\tau \tau$ final state in proton-proton collisions at $\sqrt{s}=13 \mathrm{TeV}$, JHEP 09 (2018)007.

[19] CMS Collaboration, Search for beyond the standard model Higgs bosons decaying into a b $\bar{b}$ pair in pp collisions at $\sqrt{s}=13 \mathrm{TeV}$, JHEP 08 (2018) 113.

[20] S. Patel, E. Fuchs, S. Liebler, G. Weiglein, CP-violating Effects on MSSM Higgs Searches, Acta Phys. Polon. Supp. 11 (2018) 223

[21] CMS Collaboration, Search for an exotic decay of the Higgs boson to a pair of light pseudoscalars in the final state with two $b$ quarks and two $\tau$ leptons in proton-proton collisions at $\sqrt{s}=13 \mathrm{TeV}$, PLB 785 (2018) 462. 
[22] CMS Collaboration, Search for an exotic decay of the Higgs boson to a pair of light pseudoscalars in the final state of two muons and two $\tau$ leptons in proton-proton collisions at $\sqrt{s}=13 \mathrm{TeV}$, JHEP 11 (2018) 018.

[23] CMS Collaboration, Search for a standard model-like Higgs boson in the mass range between 70 and $110 \mathrm{GeV}$ in the diphoton final state in proton-proton collisions at $\sqrt{\mathrm{s}}=8$ and $13 \mathrm{TeV}$, arXiv:1811.08459, submitted to PLB.

[24] ATLAS Collaboration, Search for resonances in the 65 to $110 \mathrm{GeV}$ diphoton invariant mass range

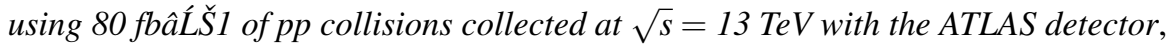
ATLAS-CONF-2018-025.

[25] ATLAS Collaboration, Search for heavy resonances decaying into $W W$ in the ev $\mu v$ final state in pp collisions at $\sqrt{s}=13 \mathrm{TeV}$ with the ATLAS detector, EPJ C78 (2018) 24.

[26] CMS Collaboration, Search for a new scalar resonance decaying to a pair of $Z$ bosons in proton-proton collisions at $\sqrt{s}=13 \mathrm{TeV}$, JHEP 06 (2018) 127.

[27] ATLAS Collaboration, Combination of searches for Higgs boson pairs in pp collisions at 13 TeV with the ATLAS experiment, ATLAS-CONF-2018-043.

[28] CMS Collaboration, Combination of searches for Higgs boson pair production in proton-proton collisions at $\sqrt{s}=13 \mathrm{TeV}$, arXiv:1811.09689, submitted to PRL.

[29] ATLAS Collaboration, Summary plots from the ATLAS Higgs physics group, https: / / at las . web. cern. ch/At las / GROUPS / P HYS ICS / Comb i nedSummaryPlots / HIGGS/.

[30] CMS Collaboration, Summary results of high mass BSM Higgs searches using CMS run-I data, CMS-PAS-HIG-16-007.

[31] CMS Collaboration, Projection of the Run 2 MSSM $\mathrm{h} \rightarrow \tau \tau$ limits for the High-Luminosity LHC, CMS-PAS-FTR-18-017.

[32] N. Craig, J. Hajer, Y. Li, T. Liu, H. Zhang, Heavy Higgs bosons at low $\tan \beta$ : from the LHC to 100 $\mathrm{TeV}$, JHEP 01 (2017) 018 